\title{
Variability in airway inflammation, symptoms, lung function and reliever use in asthma: anti-inflammatory reliever hypothesis and STIFLE study design
}

\author{
Tim Harrison ${ }^{1}$, Ian D. Pavord ${ }^{2}$, James D. Chalmers ${ }^{3}$, Glenn Whelan ${ }^{4}$, \\ Malin Fagerås ${ }^{5}$, Annika Rutgersson ${ }^{5}$, Laura Belton ${ }^{6}$, Shahid Siddiqui ${ }^{4}$ and \\ Per Gustafson ${ }^{5}$
}

Affiliations: ${ }^{1}$ Nottingham NIHR Biomedical Research Unit, University of Nottingham, Nottingham, UK. ${ }^{2}$ Respiratory Medicine Unit and Oxford Respiratory NIHR BRC, Nuffield Dept of Medicine, University of Oxford, Oxford, UK. ${ }^{3}$ Scottish Centre for Respiratory Research, University of Dundee, Ninewells Hospital and Medical School, Dundee, UK. ${ }^{4}$ AstraZeneca, Gaithersburg, MD, USA. ${ }^{5}$ AstraZeneca, Gothenburg, Sweden. ${ }^{6}$ AstraZeneca, Cambridge, UK.

Correspondence: Tim Harrison, Faculty of Medicine and Health Sciences, University of Nottingham, Nottingham, NG5 1PB, UK. E-mail: tim.harrisonanottingham.ac.uk

ABSTRACT Asthma is a chronic inflammatory airway disease. Increase in airway inflammation is hypothesised to contribute to worsening of asthma symptoms and deterioration in lung function, resulting in the use of reliever medication. Short-acting $\beta_{2}$-agonists only treat the symptoms, whereas an anti-inflammatory reliever is believed to treat both symptoms and the underlying inflammation, thereby arresting the progression to an exacerbation. As-needed budesonide/formoterol as an anti-inflammatory reliever reduces the risk of severe exacerbations. However, supporting mechanistic evidence has not yet been described, specifically the temporal dynamics of parameters including airway inflammation, over time and during asthma worsening.

The STIFLE study aims to characterise daily variability in airway inflammation, symptoms, lung function and reliever use in people with asthma. This phase IV, open-label, parallel-group, multicentre, exploratory study will enrol 60-80 adult patients with asthma receiving low- or medium-dose inhaled corticosteroids/ long-acting $\beta_{2}$-agonists (EudraCT identifier number 2018-003467-64). Participants will be randomised 1:1 to either as-needed budesonide/formoterol dry-powder inhaler or salbutamol reliever for 24 weeks, in addition to their maintenance therapy. Daily data will be captured for fractional exhaled nitric oxide, spirometry, asthma symptoms and medication use using devices connected to a smartphone via the STIFLE application. STIFLE will thereby enable not only characterisation of the variability of airway inflammation and clinical outcomes in relation to asthma worsening, but also elucidate the effect of as-needed budesonide/formoterol on airway inflammation against a background of daily maintenance therapy.

@ERSpublications

Using novel, home-based tools to generate new insights into the link between inflammation and asthma attacks, the STIFLE study aims to increase understanding of the mechanisms behind the benefits of budesonide/formoterol anti-inflammatory reliever therapy https://bit.ly/33X1wJU

Cite this article as: Harrison T, Pavord ID, Chalmers JD, et al. Variability in airway inflammation, symptoms, lung function and reliever use in asthma: anti-inflammatory reliever hypothesis and STIFLE study design. ERJ Open Res 2020; 6: 00333-2019 [https://doi.org/10.1183/23120541.003332019].

This study is registered at https://eudract.ema.europa.eu/ with identifier number 2018-003467-64.

This article has supplementary material available from openres.ersjournals.com. Qualified researchers can request access to anonymized individual patient-level data from AstraZeneca group of companies sponsored clinical trials via the request portal. All request will be evaluated as per the AZ disclosure commitment: https://astrazenecagrouptrials. pharmacm.com/ST/Submission/Disclosure.

Received: 28 Nov 2019 | Accepted after revision: 24 March 2020

Copyright $\odot$ ERS 2020. This article is open access and distributed under the terms of the Creative Commons Attribution Non-Commercial Licence 4.0. 


\section{Introduction and study rationale}

Asthma is a chronic inflammatory disease of the airways [1]. The inflammation is variable in terms of intensity and is heterogeneous, with well-characterised subtypes that have different responses to therapy $[1,2]$. In parallel, variability in lung function, asthma symptoms and reliever medication use are commonly observed over days and weeks [3-5]. Often, these periods, beyond baseline day-to-day or week-to-week variability, are interspersed with significant exacerbations of symptoms [6], necessitating increased reliever use and, potentially, the use of oral corticosteroids [7].

Treatment with inhaled corticosteroids (ICS) is the cornerstone of asthma management [7] and treatment strategies targeted at inflammation (via maintenance of a normal airway eosinophil count) have been shown to reduce the risk of severe exacerbations compared with strategies based on assessment of symptoms, lung function and use of rescue treatment [8]. However, many patients, regardless of the underlying disease severity, are not adherent with such treatment and rely on short-acting $\beta_{2}$-agonists (SABAs) for rapid relief of asthma symptoms [9]. Such over-reliance on SABAs is a concern since their excessive use may worsen asthma control and increase the risk of fatal or near-fatal asthma [10, 11]. A SABA does not address the underlying inflammation and increases the risk of exacerbations [12]. In addition, even in patients whose disease is apparently controlled and who are adherent to maintenance medication, exacerbations may still occur [9]. This suggests that, in such a variable condition, patients' requirement for anti-inflammatory therapy varies and, in turn, represents an argument in favour of providing increased doses of ICS when symptoms worsen. To this end, a combination of budesonide/ formoterol (ICS/fast-onset but long-acting $\beta_{2}$-agonist (LABA)) as maintenance and reliever therapy has been studied in several randomised controlled trials in patients with moderate-to-severe asthma [5, 13-16]. Results from these trials have demonstrated that as-needed use of budesonide/formoterol as an anti-inflammatory reliever in addition to daily maintenance therapy is highly effective in reducing the risk of severe exacerbations, with up to a $47 \%$ reduction versus comparator regimens that utilise a SABA as a reliever $[5,13,16]$. In addition, as-needed budesonide/formoterol has been evaluated as a treatment option for patients with mild asthma, as assessed in the SYGMA (Symbicort Given as Needed in Mild Asthma) programme [17, 18], the PRACTICAL (Personalised Asthma Combination Therapy: with Inhaled Corticosteroid and Fast Onset Long-Acting Beta Agonist) study [19] and the Novel START (Novel Symbicort Turbuhaler Asthma Reliever Therapy) study [20]. In the latter study, the risk of severe exacerbations was lower in the as-needed budesonide/formoterol group compared with as-needed SABA, a finding that was paralleled by a reduction in exhaled nitric oxide fraction $\left(F_{\mathrm{ENO}}\right)$, consistent with an effect of as-needed budesonide/formoterol on airway inflammation [20].

\section{Hypothesis of the STIFLE study: use of anti-inflammatory reliever therapy in the "window of opportunity"}

The benefits of anti-inflammatory reliever therapy in reducing the risk of exacerbations are hypothesised to be due to additional anti-inflammatory ICS therapy received at pivotal times [21], specifically during the "window of opportunity" when inflammation-driven symptoms start to worsen (figure 1 and supplementary video) [22, 23]. This is based on the work of TATTERSFIELD et al. [23], who evaluated all severe exacerbations that occurred in the FACET (Formoterol and Corticosteroids Establishing Therapy) study. Exacerbations were characterised by a gradual fall in peak expiratory flow (PEF) over 5-7 days, followed by a more rapid fall over 2-3 days prior to an exacerbation. Furthermore, the change in PEF occurred in parallel with an increase in symptoms and the use of SABA reliever, suggesting that a 2-3-day window of opportunity exists when patients experience deteriorating symptoms and lung function but have not yet deteriorated to the stage of requiring oral corticosteroids. Indeed, a post hoc analysis of $F_{\mathrm{ENO}}$ data from the CHARISM (Childhood Asthma Respiratory Inflammatory Status Monitoring) study showed an increase in $F_{\mathrm{ENO}} \sim 10$ days before a moderate exacerbation, with a significant increase 3 days beforehand [24]. Together, these findings highlight an early opportunity to adjust treatment with an increase in ICS to target the underlying flare-up of airway inflammation, thereby arresting progression to exacerbation. In addition, against a background of budesonide/formoterol maintenance therapy, as-needed formoterol demonstrated better exacerbation control than a SABA [14], likely due to a stabilising effect on airway smooth muscle. Furthermore, LABAs may be particularly active against noneosinophilic exacerbations [25].

However, supporting mechanistic evidence has not yet been described for as-needed budesonide/ formoterol, specifically the nature of the variations and temporal dynamics (patterns or trends with respect to time) of airway inflammation, symptoms, lung function and reliever use, both over time and during periods of symptom worsening. Consequently, the STIFLE study (EudraCT identifier number 2018-003467-64) has been designed to characterise the day-to-day relationship between such parameters, and specifically in relation to worsening of symptoms in patients with asthma receiving as-needed 

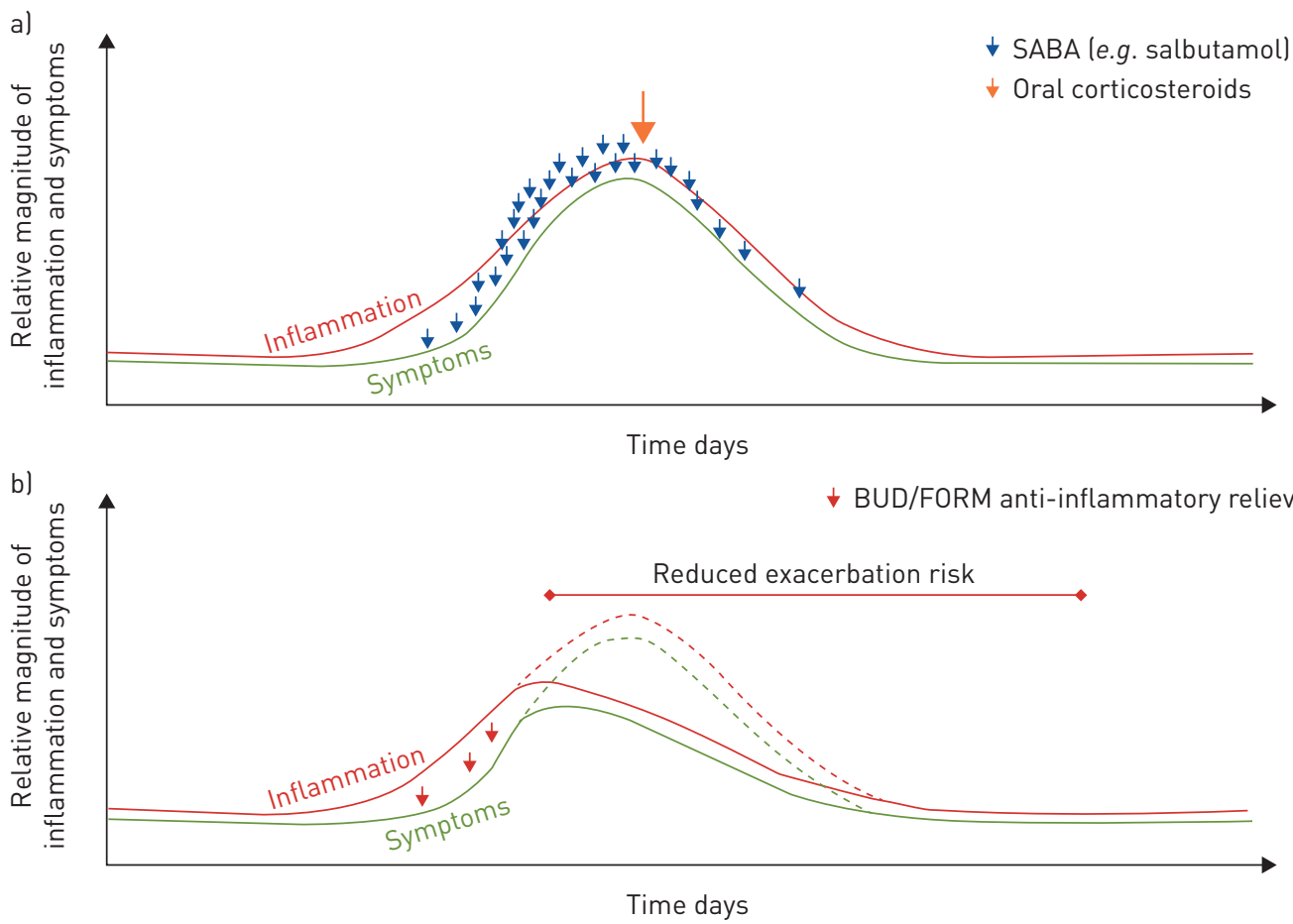

FIGURE 1 Proposed hypothesis of the mechanism of al short-acting $\beta_{2}$-agonist (SABA) reliever and b) as-needed budesonide (BUD)/formoterol (FORM) as anti-inflammatory reliever therapy during worsening of asthma symptoms that precedes an exacerbation. The effects of FORM are likely to be more complex and are not presented in the graphs. SABA treats only symptoms and not the underlying inflammation. Anti-inflammatory reliever therapy treats both symptoms and the underlying inflammation. Dashed lines indicate asthma worsening in patients with SABA reliever use.

budesonide/formoterol anti-inflammatory reliever or a SABA, against a background of budesonide/ formoterol maintenance therapy.

\section{Design of the STIFLE study}

STIFLE is a phase IV, randomised, active-comparator, open-label, parallel-group, multicentre, exploratory, 24 -week study in adult asthma patients ( $\geqslant 18$ years of age) receiving Global Initiative for Asthma 2018 [26] step 3 or step 4 treatment (low- or medium-dose ICS/LABA only). The study aims to characterise variability in inflammation $\left(F_{\mathrm{ENO}}\right)$, symptoms, lung function and reliever use, on a daily basis, over time (primary objective) and in the period surrounding an event (secondary objective). Events include severe exacerbation, Composite Endpoint for Exacerbations (CompEx) [27], a single day with six or more occasions of reliever medication use and $F_{\mathrm{ENO}}>50 \mathrm{ppb}$ (table 1). Key inclusion and exclusion criteria are shown in table 2. This study will consist of a screening visit (visit 1), a 2-week run-in period, a baseline visit (visit 2) and a 24-week treatment period (visits 3 and 4) (figure 2). During the run-in period, patients will receive maintenance budesonide/formoterol $100 / 6 \mu \mathrm{g}$ or $200 / 6 \mu \mathrm{g}$ dry-powder inhaler, two inhalations twice daily (based on ongoing low- or medium-dose ICS/LABA, respectively, at study entry) and SABA reliever (salbutamol $100 \mu \mathrm{g}$ ) as needed. At visit 2 (baseline visit, week 1), eligible patients will be randomised 1:1 (stratified by ongoing low- or medium-dose of ICS/LABA at study entry) to receive maintenance budesonide/formoterol $(100 / 6 \mu \mathrm{g}$ or $200 / 6 \mu \mathrm{g}$, two inhalations twice daily) with either as-needed budesonide/formoterol (same dose as maintenance) or salbutamol $(100 \mu \mathrm{g})$ as reliever for 24 weeks. Additional visits will be scheduled if a patient meets any of the criteria for an event during the treatment period, including the first occurrence of a severe exacerbation, symptom worsening (decrease in $\mathrm{PEF}$, increase in reliever use or asthma symptoms) or a single day with six or more occasions of reliever medication use. Informed consent will be obtained from all patients prior to initiation of study-related procedures, sampling and analysis.

All patients will be provided with a kit containing a smartphone and four devices (a $F_{\text {ENO }}$ monitoring device (Vivatmo Me; Bosch, Waiblingen, Germany), a spirometer (Spirobank Smart; MIR, Rome, Italy) and two inhaler sensors (SmartTurbo and SmartTouch; Adherium, Auckland, New Zealand)) connected to the 
TABLE 1 STIFLE study objectives and endpoints

Objectives

\section{Primary}

To characterise descriptively ${ }^{\#}$ the relationship between airway inflammation, asthma symptoms, lung function and reliever use, in the two treatment arms measured daily over 24 weeks of treatment

\section{Secondary}

To characterise descriptively ${ }^{\#}$ airway inflammation, asthma symptoms, lung function and reliever use profiles surrounding an event in the two treatment arms

\section{Exploratory}

To describe patterns of inflammatory biomarkers in the two treatment arms at the start of an event ${ }^{+}$land every 4 days up to 12 days)

To characterise descriptively the daily variations in nasal biomarker measures during the first 30 days of treatment in the two treatment arms

\section{Endpoints}

Individual patient profiles of daily variations over time in $F_{\text {ENO }}$ (morning), asthma symptom scores (morning and evening), PEF and $\mathrm{FEV}_{1}$ (morning and evening), and reliever use

Individual patient profiles of daily variations over time in $F_{\mathrm{ENO}}$ (morning), asthma symptom scores (morning and evening), PEF and $\mathrm{FEV}_{1}$ (morning and evening), and reliever use between 14 days prior and 28 days after an event. Events include severe exacerbation?" CompEx (full criteria) [26], single day (in $24 \mathrm{~h}$ ) with six or more occasions of reliever medication use and $F_{\text {ENO }}>50 \mathrm{ppb}$

Individual patient profiles of inflammation biomarkers (blood biomarkers including eosinophils, EDN and CRP; sputum and nasal biomarkers) at the start of an event (and every 4 days up to 12 days)

Individual patient profiles of daily variations over time in nasal biomarkers during the first 30 days of treatment (subset of 30 patients)

Severe exacerbation events will be recorded, as they occur, on a case report form. Other events will be retrospectively defined and analysed based on the data collected during the study. $F_{E N O}$ : exhaled nitric oxide fraction; PEF: peak expiratory flow; FEV 1 : forced expiratory volume in $1 \mathrm{~s}$; CompEx: Composite Endpoint for Exacerbations; EDN: eosinophil-derived neurotoxin; CRP: C-reactive protein. ${ }^{\#}$ : this study has not been designed or powered to test any statistical hypothesis relating to a difference between the treatment arms; instead, airway inflammation, symptoms, lung function and reliever use profiles will be assessed descriptively and visually and will be modelled for each patient individually and by treatment, both over the 24-week period and during the time surrounding the various events. ๆ: use of systemic corticosteroids for $\geqslant 3$ days, an emergency department visit due to asthma that requires systemic corticosteroids or inpatient hospitalisation due to asthma. ${ }^{+}$: events of interest include the first occurrence of either a severe exacerbation, symptom worsening (deterioration in two or more variables for two or more consecutive days: $\geqslant 15 \%$ decrease in PEF, $\geqslant 1.5$ times increase in inhalations of reliever medication or $\geqslant 1$ increase in asthma symptom score lor the absolute maximum score of 3), in the morning or evening, as compared with baseline), or a single day (in $24 \mathrm{~h}$ ) with six or more occasions of reliever use.

TABLE 2 Key inclusion and exclusion criteria

\section{Inclusion criteria}

Male and/or female patients aged $\geqslant 18$ years

Physician-diagnosed asthma for $\geqslant 6$ months prior to visit 1

Use of low- or medium-dose ICS/LABA ${ }^{\#}$ for $\geqslant 3$ months prior to visit 1

Worsening of asthma symptoms requiring overuse $^{\text {ๆ }}$ of reliever medication at least once within 30 days prior to visit 1

Patient-reported history of one or more severe asthma exacerbations requiring treatment with systemic corticosteroids during the 12 months prior to visit 1 , except in the previous 30 days

Ability to perform $F_{\text {ENO }}$ and spirometry assessments at home and complete an asthma symptom diary on a regular basis

\section{Exclusion criteria}

Patients with any significant disease or disorder or evidence of drug/substance abuse that would pose a risk to patient safety, interfere with study conduct or impact study results

Any asthma worsening requiring a change in treatment other than the prescribed reliever medication within 30 days prior to visit 1

Medical history of life-threatening asthma, including intubation and admission to an intensive care unit

Medical conditions (except allergic rhinitis) or medications (except ICS) that may influence $F_{\text {ENO }}$

Concurrent respiratory disease (e.g. cystic fibrosis, idiopathic pulmonary fibrosis or pulmonary arterial hypertension)

Acute upper or lower respiratory infections requiring antibiotics or antiviral medication within 30 days prior to visit 1 or during screening/run-in period

A severe exacerbation (resulting in $\geqslant 3$ days of oral corticosteroids lor one depot intramuscular injection of glucocorticosteroid), an urgent care or emergency room visit resulting in treatment with systemic corticosteroids or an inpatient hospitalisation due to asthmal within 30 days prior to screening

Any disease, other than asthma, or procedure that may necessitate the use of oral/systemic corticosteroids during the treatment period

Current tobacco smoking or a history of smoking for $\geqslant 10$ pack-years

ICS: inhaled corticosteroid; LABA: long-acting $\beta_{2}$-agonist; $F_{\text {ENO }}$ : exhaled nitric oxide fraction. \#: low ICS doses (daily): beclometasone dipropionate hydrofluoroalkane (HFA) $(100-200 \mu \mathrm{g})$, budesonide dry-powder inhaler (DPI) $(200-400 \mu \mathrm{g})$, ciclesonide HFA (80-160 $\mu \mathrm{g}$ ), fluticasone furoate DPI $(100 \mu \mathrm{g})$, fluticasone propionate DPI or HFA $(100-250 \mu \mathrm{g})$, mometasone furoate $(110-220 \mu \mathrm{g})$ and triamcinolone acetonide (400-1000 $\mu \mathrm{g}$ ); medium ICS doses (daily): beclometasone dipropionate HFA (>200-400 $\mu \mathrm{g}$ ), budesonide DPI (>400-800 $\mu \mathrm{g}$ ), ciclesonide HFA $(>160-320 \mu \mathrm{g})$, fluticasone propionate DPI or HFA $(>250-500 \mu \mathrm{g})$, mometasone furoate $(>220-440 \mu \mathrm{g})$ and triamcinolone acetonide $(>1000-2000 \mu \mathrm{g}$ ). "?: overuse was defined as more than the standard use of reliever for individual patients. 


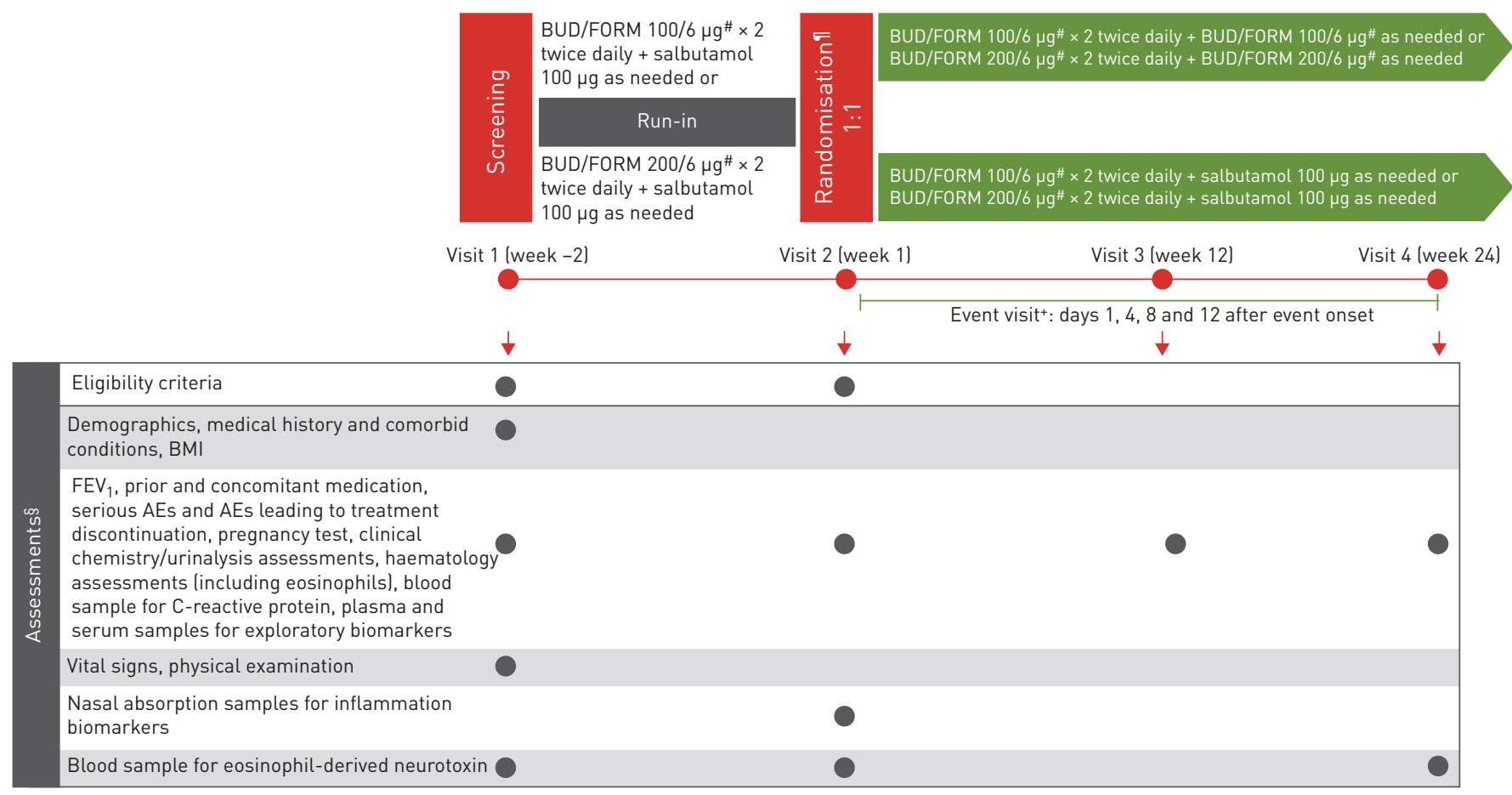

FIGURE 2 Study design. BMI: body mass index. \#: patients on low-dose inhaled corticosteroid (ICS)/long-acting $\beta_{2}$-agonist (LABA) at study entry will receive budesonide (BUD)/formoterol (FORM) 100/6 $\mu \mathrm{g}$ and those on medium-dose ICS/LABA at study entry will receive BUD/FORM 200/6 $\mu \mathrm{g}$. I: randomisation criteria are adherence (completed $\geqslant 80 \%$ of asthma assessments (exhaled nitric oxide fraction ( $F_{\text {ENO }}$ ) and spirometry measurements, and completing the asthma symptom diaryl at home during the run-in period) and reliever medication use (used reliever medication for 2-8 days out of the last 10 days of the run-in period). ${ }^{+}$: event visit assessments are prior and concomitant medication (days 1 and 12), serious adverse events (AEs) and AEs leading to treatment discontinuation, clinical chemistry, urinalysis, haematology (including eosinophils), spontaneous sputum for inflammatory biomarkers, nasal absorption samples for inflammatory biomarkers, blood sample leosinophil-derived neurotoxin and C-reactive protein), plasma and serum samples for exploratory biomarkers. $\S_{\text {: }}$ daily assessments at home are $F_{\text {ENo, }}$ peak expiratory flow (PEF) and forced expiratory volume in $1 \mathrm{~s}\left(\mathrm{FEV}_{1}\right)$, and completing the asthma symptom diary before taking the study medication in the morning; and PEF and $\mathrm{FEV}_{1}$, and completing the asthma symptom diary before taking the study medication in the evening.

smartphone via the STIFLE application (AstraZeneca, Cambridge, UK) and will receive training on its use. Daily, at home, patients will perform $F_{\mathrm{ENO}}$ measurements (morning) and spirometry assessments (PEF and forced expiratory volume in $1 \mathrm{~s}\left(\mathrm{FEV}_{1}\right)$, morning and evening) and complete an asthma symptom diary that includes reliever medication use (morning and evening). Data from these four devices and an embedded asthma symptom diary will be recorded in the STIFLE application through an integrated approach (figure 3). $\sim 30$ patients who agree to participate in a nasal biomarker substudy of STIFLE will be shown how to collect a nasal absorption sample (synthetic absorptive matrix (SAM) [28]), and will be provided with a kit and instructions for collecting the samples at home. These patients will collect daily morning nasal samples for the first 30 days of randomised treatment for the evaluation of inflammatory biomarkers.

At all clinic visits, $\mathrm{FEV}_{1}$ will be assessed (by clinic spirometry equipment), safety (adverse events) will be evaluated, and blood samples will be collected for haematology (including eosinophils), clinical chemistry and C-reactive protein analyses. 60-80 patients will be randomised from three sites in the UK to achieve $\geqslant 50$ patients who will complete the 24 -week study. It is anticipated that half of these patients will meet the criteria for an event.

\section{A short review: $F_{\text {ENO }}$}

Daily measurement of $F_{\mathrm{ENO}}$ is an important methodological component of the STIFLE study. $F_{\mathrm{ENO}}$ is a simple, noninvasive, quantitative, highly sensitive and safe measure of airway inflammation [29]. $F_{\mathrm{ENO}}$ is a surrogate marker for eosinophilic airway inflammation and is used to determine the potential need for and likelihood of response to ICS, to optimise the doses of anti-inflammatory medications, and to unmask poor adherence to ICS [29]. A strength of the STIFLE study is that we will measure $F_{\mathrm{ENO}}$ concentrations daily, against a background of maintenance ICS therapy, to determine if variability in $F_{\mathrm{ENO}}$ around the time of an exacerbation can be detected and, therefore, whether it is a useful biomarker of type 2 inflammation in a cohort of treated patients with moderate asthma. 

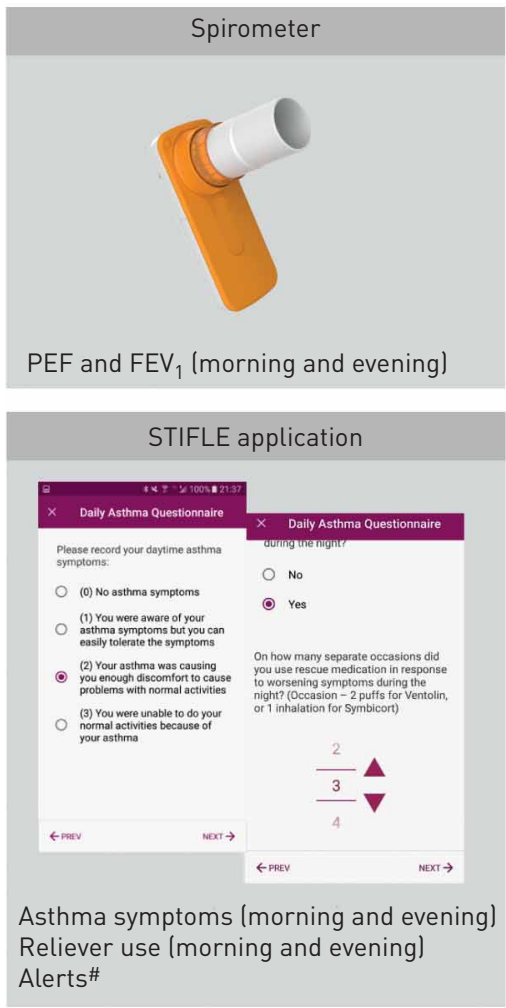
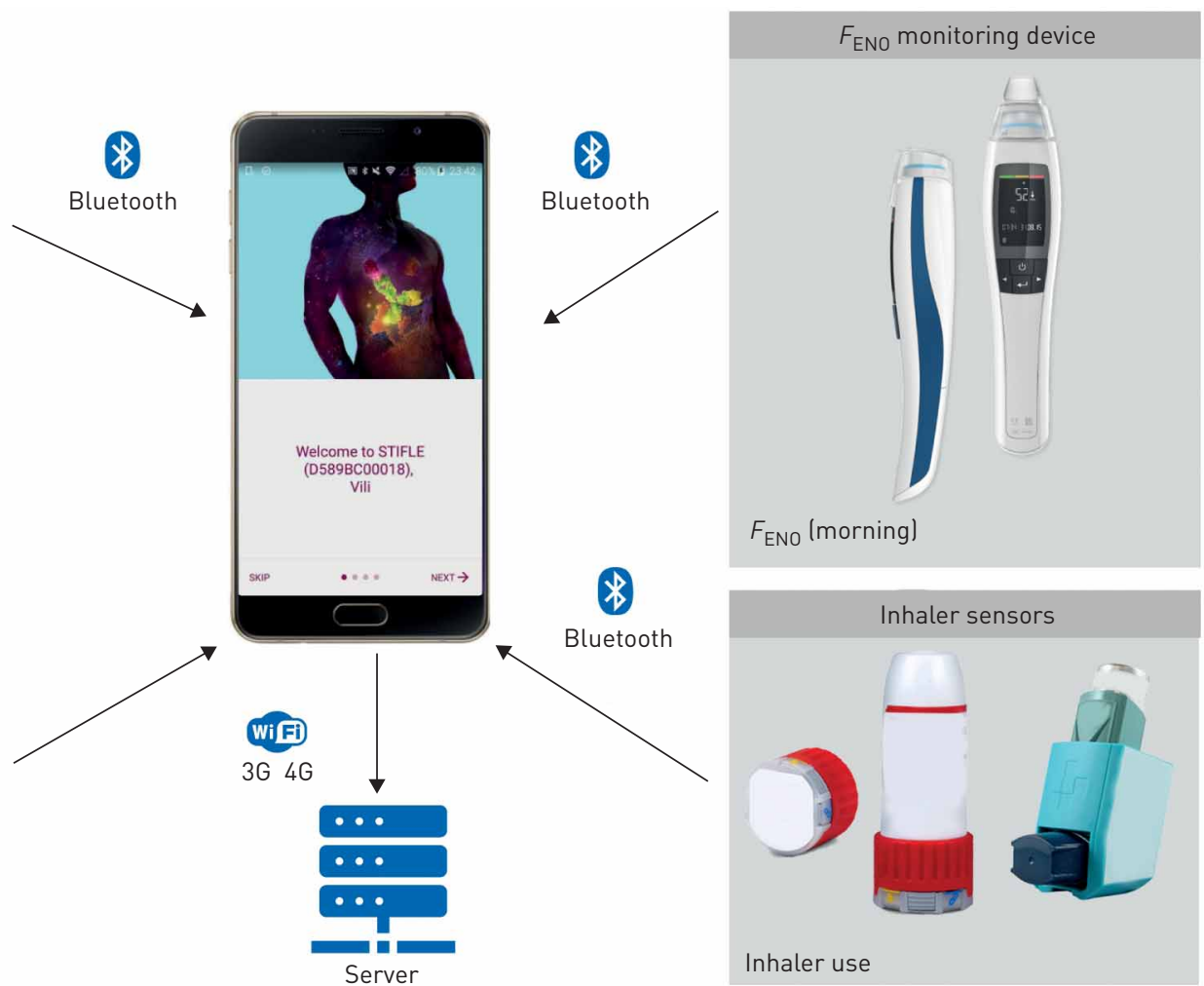

FIGURE 3 Integrated approach to data collection. PEF: peak expiratory flow; FEV ${ }_{1}$ : forced expiratory volume in $1 \mathrm{~s}$; $F_{\text {ENO }}$ : exhaled nitric oxide fraction. ": the patient and the investigator will receive alerts from the STIFLE system regarding event visits (in relation to the exploratory objective event) due to symptom worsening (based on peak expiratory flow (PEF), reliever use and symptom score criteria) or due to a single day with six or more occasions of reliever medication use. Patients will also be instructed to contact the study site to arrange an event visit if they experience a severe exacerbation. Patients and investigators will be blinded to all data collected from these devices. Asthma symptoms during the night and day will be recorded by the patient twice daily in the asthma symptom diary, according to the following scoring system: 0 , no asthma symptoms; 1, "you are aware of your asthma symptoms, but you can easily tolerate the symptoms"; 2, "your asthma is causing you enough discomfort to cause problems with normal activities (or with sleep)"; 3, "you are unable to do your normal activities (or to sleep) because of your asthma".

\section{How will the STIFLE study help understand the day-to-day relationship between airway inflammation, symptoms and lung function in patients with asthma?}

To our knowledge, STIFLE will be the first study specifically designed to investigate daily airway inflammatory patterns and associated variability in symptoms, lung function and reliever medication use in patients with asthma. An increase in airway inflammation is thought to contribute to worsening of asthma symptoms and deterioration in lung function, requiring the use of reliever medication. However, the time perspective of various parameters before and after asthma worsening is largely unknown, including the order of appearance, magnitude and timeframe in relation to worsening. Therefore, the STIFLE study will help clarify these differences and provide an understanding of the temporal dynamics of these parameters, and specifically in relation to asthma worsening.

High "as-needed" reliever use is a robust measure of asthma instability [22]. Results from an exploratory analysis of two randomised controlled trials reported that budesonide/formoterol maintenance and anti-inflammatory reliever therapy reduced the incidence of high reliever use episodes (more than six inhalations a day) and exacerbation burden immediately following these high-use episodes compared with ICS/LABA maintenance plus SABA reliever therapy [22]. Consequently, STIFLE will also help establish whether the hypothesised mechanistic benefit of as-needed budesonide/formoterol as anti-inflammatory reliever relates to the timely and rapid suppression of airway inflammation (and related symptoms) by ICS during the window of opportunity [21, 22], as compared with only symptom relief provided by a SABA (figure 1). The concentration of ICS in lung tissue has been shown to decline over time (between maintenance doses) [30], with concentrations being $\sim 10 \%$ of peak values within $6 \mathrm{~h}$ of inhalation; thus, as-needed ICS may help restore concentrations when the levels of ICS are suboptimal between scheduled maintenance doses [14]. The importance of increasing ICS dosing frequency is supported by the results of a crossover study in 34 adult asthma outpatients where greater efficacy was consistently observed with 
budesonide four times daily dosing compared with two times daily dosing, while providing the same total daily dose [31]. In addition to the timely increase in ICS dose, the rapid onset of action of ICS is of particular importance, with a wealth of evidence supporting the rapid anti-inflammatory effect of inhaled budesonide (with or without formoterol) [32-35]. Indeed, results from a randomised controlled trial in 41 patients with stable asthma reported a significant reduction in sputum eosinophils and an improvement in airway responsiveness within $6 \mathrm{~h}$ of budesonide inhalation [32]. Furthermore, a systemic anti-inflammatory effect on circulating lymphocytes within $30 \mathrm{~min}$ of inhalation with budesonide/ formoterol has been demonstrated [34]. Additionally, budesonide/formoterol has been reported to prevent asthma deterioration and significantly improve baseline $\mathrm{FEV}_{1}$ at $30 \mathrm{~min}$ following a low-dose allergen exposure in patients with intermittent allergic asthma [35].

Notably, the STIFLE study will incorporate several important methodological components. The main outcomes will be based on daily measurements, including local airway inflammation as measured by $F_{\mathrm{ENO}}$ concentrations taken at home. To date, very few studies have evaluated $F_{\mathrm{ENO}}$ in the setting of acute exacerbations and those that have report varying results [36-38]. Results from a cross-sectional study in a paediatric emergency department reported that $F_{\mathrm{ENO}}$ concentrations during an exacerbation did not correlate with asthma severity and had limited utility in the management of acute asthma [36]. In contrast, in adult patients with moderate-to-severe asthma, a significant increase in $F_{\mathrm{ENO}}$ concentrations during exacerbations compared with baseline in certain patients was reported [37]. In a sample of emergency department patients with acute exacerbations of asthma, $F_{\mathrm{ENO}}$ measurements were poorly reproducible and did not correlate with asthma severity [38]. However, a significant increase in $F_{\text {ENO }}$ was reported during 3 days before until 4 days after moderate exacerbations in children with asthma [24]. The results from STIFLE are therefore expected to contribute to this knowledge base. Although $F_{\text {ENO }}$ measurements have been implemented in many asthma trials, including studies utilising daily, domiciliary methods for capturing $F_{\mathrm{ENO}}[39,40]$, a novel aspect of this study is that daily home assessments of $F_{\mathrm{ENO}}$ will be used to determine variability and to compare with other parameters (lung function, reliever use and symptoms) in a real-time environment to assess maintenance plus SABA versus maintenance plus anti-inflammatory reliever therapy over 6 months.

A potential limitation of using $F_{\mathrm{ENO}}$ measurements is that rhinitis and allergic comorbidities in patients with asthma may also lead to changes in $F_{\mathrm{ENO}}$ levels [41]. However, in addition to $F_{\mathrm{ENO}}$, other biomarkers of inflammation, such as sputum and blood eosinophils, eosinophil-derived neurotoxin, and nasal

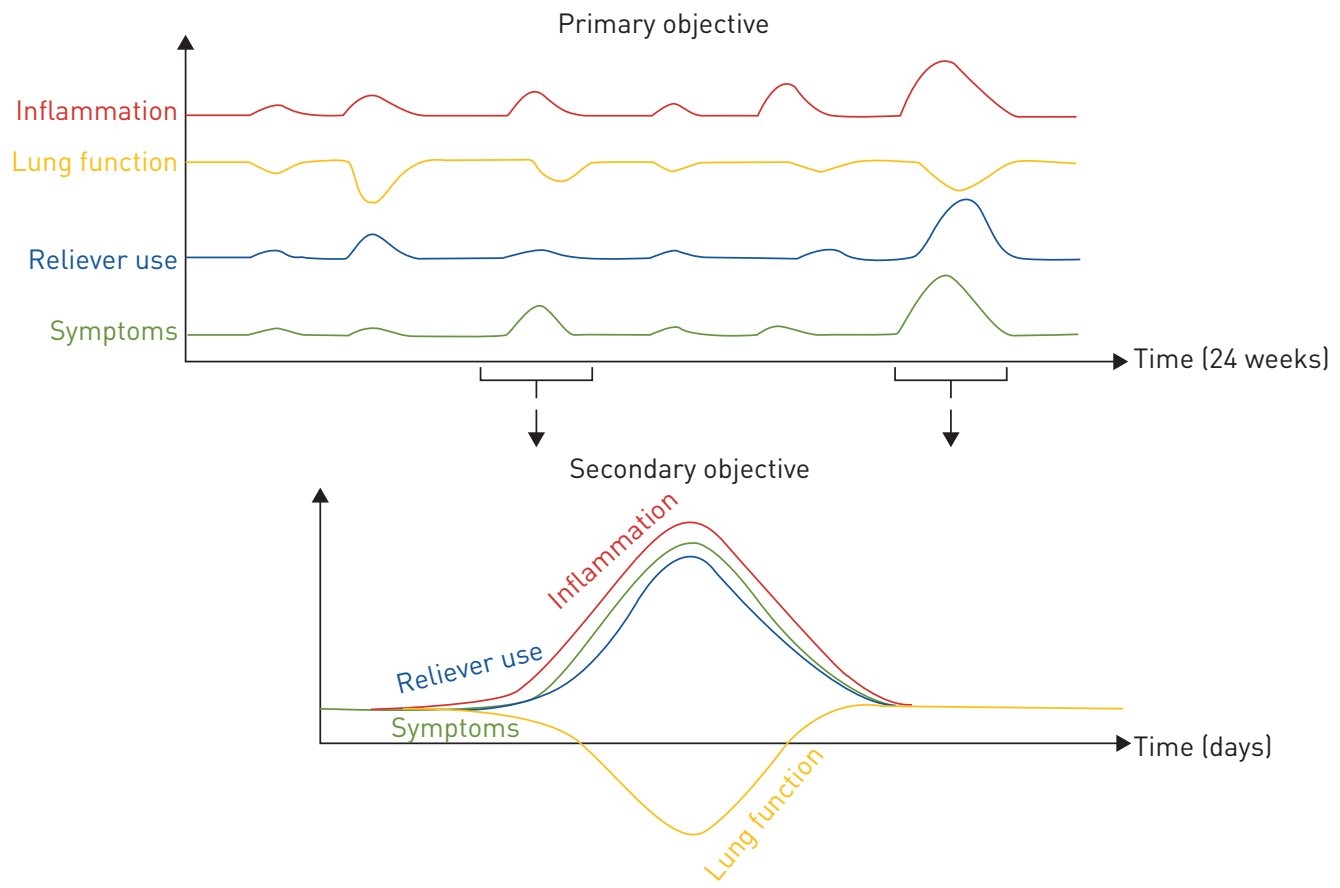

FIGURE 4 Examination of endpoints over 24 weeks and specifically in relation to an event. The graphs are for representation only. The variables may or may not show parallel changes at a specific time point. Results from the STIFLE study would potentially provide an understanding of the temporal dynamics of these parameters. 
absorption biomarkers, will also be measured to explore patterns of inflammation. As patients will be performing $F_{\mathrm{ENO}}$ and spirometry assessments at home, it is necessary that they use these devices correctly. Spirometers can be difficult to use in the community and challenging to interpret. However, all patients are trained on device use. Moreover, there is a precedence for the use of the spirometer in the home environment $[42,43]$.

Another important feature of the study design of STIFLE is the examination of the temporal dynamics of clinical parameters and local inflammation in relation to an event (figure 4), which may provide insights into the inflammatory mechanism of worsening symptoms and lung function and highlight the benefits of an anti-inflammatory reliever compared with a SABA reliever. Notably, STIFLE will include patients on a background of ICS/LABA maintenance since variability in lung function, asthma symptoms and reliever medication use are also observed in patients receiving such therapy [5, 9], many of whom continue to be at risk of exacerbations even when adherent to therapy [44].

STIFLE will also be among one of the first prospective studies to use CompEx, an extended definition of asthma exacerbations that combines diary-based events (clinically relevant asthma deteriorations) with the traditional definition of severe exacerbations [27]. Since severe exacerbations are rare events, clinical trials using such events as the primary outcome measure require large sample sizes and a long study duration to evaluate treatment effects. Thus, CompEx, which mirrors results observed with the severe exacerbation-validated outcome [27], was incorporated into the STIFLE study because it allows for the evaluation of clinically relevant worsening in this clinical trial of short duration and with relatively few patients.

\section{Conclusion}

In summary, the temporal dynamics of clinical parameters and airway inflammation are largely unknown in patients with asthma, and the mechanistic evidence for the anti-inflammatory effect of as-needed budesonide/formoterol has not been described. STIFLE is expected to provide insights into the real-time day-to-day relationship between airway inflammation, lung function, asthma symptoms and reliever use in such patients, including the temporal dynamics of various parameters surrounding events. Results will further elucidate the anti-inflammatory effect of budesonide/formoterol as an anti-inflammatory reliever against a background of regular maintenance therapy.

Acknowledgements: Medical writing support was provided by Sudha Korwar of Cactus Communications (Mumbai, India), which was funded by AstraZeneca in accordance with Good Publication Practice guidelines (www.ismpp.org/ gpp3).

Conflict of interest: T. Harrison reports non-financial support from AstraZeneca for assistance with manuscript writing during the conduct of the study; grants, personal fees for advisory boards and speaker meetings, and support to attend conferences from AstraZeneca, fees for advisory boards and speaker meetings from GSK, and personal fees from Vectura, outside the submitted work. I.D. Pavord reports speaker fees, advisory board honoraria, sponsorship to attend scientific meetings and payments for organising educational events from AstraZeneca; speaker fees, advisory board honoraria and sponsorship to attend scientific meetings from Boehringer Ingelheim; a speaker fee from Aerocrine; speaker fees and advisory board honoraria from Almirall and Novartis; speaker fees, advisory board honoraria and sponsorship to attend scientific meetings from GlaxoSmithKline; advisory board honoraria from Genentech and Regeneron; speaker honoraria, payments for organising educational events and sponsorship to attend scientific meetings from Teva; speaker honoraria from Chiesi; advisory board honoraria from Sanofi, Circassia and Knopp; and funding from NIHR as a Senior Investigator, all outside the submitted work. J.D. Chalmers reports grants and personal fees from AstraZeneca during the conduct of the study; and grants and personal fees from GlaxoSmithKline, Boehringer Ingelheim, Zambon, Insmed, Grifols and Novartis, grants from Gilead, and personal fees from Napp, all outside the submitted work. G. Whelan is an employee of AstraZeneca. M. Fagerås is an employee of AstraZeneca. A. Rutgersson is an employee of AstraZeneca. L. Belton reports other from AstraZeneca, during the conduct of the study; other from AstraZeneca, outside the submitted work. S. Siddiqui has nothing to disclose. P. Gustafson is an AstraZeneca employee and shareholder.

Support statement: The STIFLE study is funded by AstraZeneca. Funding information for this article has been deposited with the Crossref Funder Registry.

\section{References}

1 National Asthma Education and Prevention Program, Third Expert Panel on the Diagnosis and Management of Asthma. Expert Panel Report 3: Guidelines for the Diagnosis and Management of Asthma. Section 2. Definition, pathophysiology and pathogenesis of asthma, and natural history of asthma. www.ncbi.nlm.nih.gov/books/ NBK7232/. Date last updated: Aug 2007. Date last accessed: July 19, 2019.

2 Robinson D, Humbert M, Buhl R, et al. Revisiting Type 2-high and Type 2-low airway inflammation in asthma: current knowledge and therapeutic implications. Clin Exp Allergy 2017; 47: 161-175.

3 Reddel H, Jenkins C, Woolcock A. Diurnal variability - time to change asthma guidelines? BMJ 1999; 319: 45-47.

4 Yawn BP. Factors accounting for asthma variability: achieving optimal symptom control for individual patients. Prim Care Respir J 2008; 17: 138-147. 
5 O’Byrne PM, Bisgaard H, Godard PP, et al. Budesonide/formoterol combination therapy as both maintenance and reliever medication in asthma. Am J Respir Crit Care Med 2005; 171: 129-136.

6 Singh AM, Busse WW. Asthma exacerbations. 2: aetiology. Thorax 2006; 61: 809-816.

7 Global Initiative for Asthma. Global strategy for asthma management and prevention. https://ginasthma.org/wpcontent/uploads/2019/06/GINA-2019-main-report-June-2019-wms.pdf. Date last updated: 2019. Date last accessed: July 19, 2019.

8 Green RH, Brightling CE, McKenna S, et al. Asthma exacerbations and sputum eosinophil counts: a randomised controlled trial. Lancet 2002; 360: 1715-1721.

9 Partridge MR, van der Molen T, Myrseth S-E, et al. Attitudes and actions of asthma patients on regular maintenance therapy: the INSPIRE study. BMC Pulm Med 2006; 6: 13.

10 Spitzer WO, Suissa S, Ernst P, et al. The use of beta-agonists and the risk of death and near death from asthma. N Engl J Med 1992; 326: 501-506.

11 Aldridge RE, Hancox RJ, Robin Taylor D, et al. Effects of terbutaline and budesonide on sputum cells and bronchial hyperresponsiveness in asthma. Am J Respir Crit Care Med 2000; 161: 1459-1464.

12 O’Byrne PM, Jenkins C, Bateman ED. The paradoxes of asthma management: time for a new approach? Eur Respir J 2017; 50: 1701133.

13 Scicchitano R, Aalbers R, Ukena D, et al. Efficacy and safety of budesonide/formoterol single inhaler therapy versus a higher dose of budesonide in moderate to severe asthma. Curr Med Res Opin 2004; 20: 1403-1418.

14 Rabe KF, Atienza T, Magyar P, et al. Effect of budesonide in combination with formoterol for reliever therapy in asthma exacerbations: a randomised controlled, double-blind study. Lancet 2006; 368: 744-753.

15 Bousquet J, Boulet LP, Peters MJ, et al. Budesonide/formoterol for maintenance and relief in uncontrolled asthma vs. high-dose salmeterol/fluticasone. Respir Med 2007; 101: 2437-2446.

16 Kuna P, Peters MJ, Manjra AI, et al. Effect of budesonide/formoterol maintenance and reliever therapy on asthma exacerbations. Int J Clin Pract 2007; 61: 725-736.

17 O'Byrne PM, FitzGerald JM, Bateman ED, et al. Inhaled Combined Budesonide-Formoterol as Needed in Mild Asthma. N Engl J Med 2018; 378: 1865-1876.

18 Bateman ED, Reddel HK, O'Byrne PM, et al. As-needed budesonide-formoterol versus maintenance budesonide in mild asthma. N Engl J Med 2018; 378: 1877-1887.

19 Hardy J, Baggott C, Fingleton J, et al. Budesonide-formoterol reliever therapy versus maintenance budesonide plus terbutaline reliever therapy in adults with mild to moderate asthma (PRACTICAL): a 52-week, open-label, multicentre, superiority, randomised controlled trial. Lancet 2019; 394: 919-928.

20 Beasley R, Holliday M, Reddel HK, et al. Controlled Trial of Budesonide-Formoterol as Needed for Mild Asthma. N Engl J Med 2019; 380: 2020-2030.

21 Barnes PJ. Scientific rationale for using a single inhaler for asthma control. Eur Respir J 2007; 29: 587-595.

22 Buhl R, Kuna P, Peters MJ, et al. The effect of budesonide/formoterol maintenance and reliever therapy on the risk of severe asthma exacerbations following episodes of high reliever use: an exploratory analysis of two randomised, controlled studies with comparisons to standard therapy. Respir Res 2012; 13: 59.

23 Tattersfield AE, Postma DS, Barnes PJ, et al. Exacerbations of asthma: a descriptive study of 425 severe exacerbations. The FACET International Study Group. Am J Respir Crit Care Med 1999; 160: 594-599.

24 van der Valk RJ, Baraldi E, Stern G, et al. Daily exhaled nitric oxide measurements and asthma exacerbations in children. Allergy 2012; 67: 265-271.

25 Jayaram L, Pizzichini MM, Cook RJ, et al. Determining asthma treatment by monitoring sputum cell counts: effect on exacerbations. Eur Respir J 2006; 27: 483-494.

26 Global Initiative for Asthma. Global strategy for asthma management and prevention. https://ginasthma.org/wpcontent/uploads/2018/04/wms-GINA-2018-report-V1.3-002.pdf. Date last updated: 2018. Date last accessed: July 19, 2019.

27 Fuhlbrigge AL, Bengtsson T, Peterson S, et al. A novel endpoint for exacerbations in asthma to accelerate clinical development: a post-hoc analysis of randomised controlled trials. Lancet Respir Med 2017; 5: 577-590.

28 Thwaites RS, Jarvis HC, Singh N, et al. Absorption of nasal and bronchial fluids: precision sampling of the human respiratory mucosa and laboratory processing of samples. J Vis Exp 2018; 131: 56413.

29 Dweik RA, Boggs PB, Erzurum SC, et al. An official ATS clinical practice guideline: interpretation of exhaled nitric oxide levels ( $F_{\mathrm{ENO}}$ ) for clinical applications. Am J Respir Crit Care Med 2011; 184: 602-615

30 Miller-Larsson A, Mattsson H, Hjertberg E, et al. Reversible fatty acid conjugation of budesonide. Novel mechanism for prolonged retention of topically applied steroid in airway tissue. Drug Metab Dispos 1998; 26: 623-630.

31 Toogood JH, Baskerville JC, Jennings B, et al. Influence of dosing frequency and schedule on the response of chronic asthmatics to the aerosol steroid, budesonide. J Allergy Clin Immunol 1982; 70: 288-298.

32 Gibson PG, Saltos N, Fakes K. Acute anti-inflammatory effects of inhaled budesonide in asthma: a randomized controlled trial. Am J Respir Crit Care Med 2001; 163: 32-36.

33 Mendes ES, Rebolledo P, Campos M, et al. Immediate antiinflammatory effects of inhaled budesonide in patients with asthma. Ann Am Thorac Soc 2014; 11: 706-711.

34 Rudiger JJ, Gencay M, Yang JQ, et al. Fast beneficial systemic anti-inflammatory effects of inhaled budesonide and formoterol on circulating lymphocytes in asthma. Respirology 2013; 18: 840-847.

35 Dahlen B, Lantz AS, Ihre E, et al. Effect of formoterol with or without budesonide in repeated low-dose allergen challenge. Eur Respir J 2009; 33: 747-753.

36 Kwok MY, Walsh-Kelly CM, Gorelick MH. The role of exhaled nitric oxide in evaluation of acute asthma in a pediatric emergency department. Acad Emerg Med 2009; 16: 21-28.

37 Gelb AF, Moridzadeh R, Singh DH, et al. In moderate-to-severe asthma patients monitoring exhaled nitric oxide during exacerbation is not a good predictor of spirometric response to oral corticosteroid. J Allergy Clin Immunol 2012; 129: 1491-1498.

38 Gill M, Walker S, Khan A, et al. Exhaled nitric oxide levels during acute asthma exacerbation. Acad Emerg Med 2005; 12: 579-586.

39 Anderson WJ, Short PM, Williamson PA, et al. Inhaled corticosteroid dose response using domiciliary exhaled nitric oxide in persistent asthma: the FENOtype trial. Chest 2012; 142: 1553-1561. 
40 Saito J, Gibeon D, Macedo P, et al. Domiciliary diurnal variation of exhaled nitric oxide fraction for asthma control. Eur Respir J 2014; 43: 474-484.

41 Gemicioglu B, Musellim B, Dogan I, et al. Fractional exhaled nitric oxide (FeNo) in different asthma phenotypes. Allergy Rhinol 2014; 5: 157-161.

42 Moor CC, Gür-Demirel Y, Wijsenbeek MS. Feasibility of a comprehensive home monitoring program for sarcoidosis. J Pers Med 2019; 9: 23.

43 Castro M, Zavod M, Rutgersson A, et al. iPREDICT: patient experience and compliance with digital technologies measuring uncontrolled asthma events and potential triggers in a 6-month study. Chest 2019; 156: A208-A210.

44 Papi A, Ryan D, Soriano JB, et al. Relationship of inhaled corticosteroid adherence to asthma exacerbations in patients with moderate-to-severe asthma. J Allergy Clin Immunol Pract 2018; 6: 1989-1998. 